Noise reduction and estimation in multiple micro-electro-mechanical inertial systems

This article has been downloaded from IOPscience. Please scroll down to see the full text article.

2010 Meas. Sci. Technol. 21065201

(http://iopscience.iop.org/0957-0233/21/6/065201)

View the table of contents for this issue, or go to the journal homepage for more

Download details:

IP Address: 128.178.82.75

The article was downloaded on 22/04/2010 at 14:27

Please note that terms and conditions apply. 


\title{
Noise reduction and estimation in multiple micro-electro-mechanical inertial systems
}

\author{
Adrian Waegli $^{1}$, Jan Skaloud ${ }^{1}$, Stéphane Guerrier ${ }^{2}$, Maria Eulàlia Parés ${ }^{3}$ \\ and Ismael Colomina ${ }^{3}$ \\ ${ }^{1}$ Swiss Federal Institute of Technology, Geodetic Engineering Laboratory, Lausanne, Switzerland \\ ${ }^{2}$ University of Geneva, Department of Statistics, Geneva, Switzerland \\ ${ }^{3}$ Institute of Geomatics, Generalitat de Catalunya and Universitat Politècnica de Catalunya, \\ Castelldefels, Spain \\ E-mail: adrian.waegli@a3.epfl.ch,jan.skaloud@epfl.ch, Stephane.Guerrier@unige.ch, \\ eulalia.pares@ideg.es and ismael.colomina@ideg.es
}

Received 17 December 2009, in final form 15 March 2010

Published 21 April 2010

Online at stacks.iop.org/MST/21/065201

\begin{abstract}
This research studies the reduction and the estimation of the noise level within a redundant configuration of low-cost (MEMS-type) inertial measurement units (IMUs). Firstly, independent observations between units and sensors are assumed and the theoretical decrease in the system noise level is analyzed in an experiment with four MEMS-IMU triads. Then, more complex scenarios are presented in which the noise level can vary in time and for each sensor. A statistical method employed for studying the volatility of financial markets $(\mathrm{GARCH})$ is adapted and tested for the usage with inertial data. This paper demonstrates experimentally and through simulations the benefit of direct noise estimation in redundant IMU setups.
\end{abstract}

Keywords: IMU, MEMS, redundancy, noise estimation, GARCH, ARMA

(Some figures in this article are in colour only in the electronic version)

\section{Introduction}

The use of redundant micro-electro-mechanical system (MEMS)-type sensors is an economically and ergonomically viable solution to improve navigation performance while enhancing sensor performance monitoring [1]. Past investigations [2] have shown that redundant inertial sensors can improve the quality of GPS (global positioning system)/INS (inertial navigation system) integration on several levels. Firstly, the noise levels of the gyros and accelerometers can be estimated directly from the data and are hence a more precise reflection of the reality. Indeed, the realistic estimation of the noise parameters in the inertial sensors is important for correct tuning of the Kalman filter (KF) used in the GPS/INS integration. Secondly, the noise level of the overall system can be reduced and defective sensors, spurious signals or sensor malfunctioning can be detected and isolated [2]. This improves the accuracy of autonomous navigation and therefore a system utilizing redundant inertial measurement unit (IMU) bridges the gaps in the GPS signals more effectively. Finally, more accurate orientation determination can be achieved with redundant IMU configurations. This represents an interesting alternative for reaching good orientation estimation with lowquality sensors yet in abundant manner.

In [3] the optimal configuration of the multiple IMU triads was studied, while in [2] their optimal insertion in the inertial navigator and GPS/INS data integrator was investigated. Here, we focus on the possibility of instantly estimating the eventually varying noise level of the inertial sensors during the processing. Indeed, it has been shown in [3] that MEMSIMUs are strongly influenced by variation of the environmental conditions (e.g. increased vibrations or temperature variations) which can introduce heteroskedasticity (i.e. if the considered sequence of random variables do not have the same finite variance) and correlation among the least-squares residuals. Due to the fact that the MEMS are manufactured in assembly lines and with micro-mechanical technology, once they are produced they are not fine-adjusted. That is, once the MEMS are produced there is no mechanical tuning of their properties; thus, the MEMS' performance is expected to be within a range 
but cannot be assured to be in a small range. Furthermore, the mechanical elements of MEMS are so small that their behavior is really dependent on environment conditions like temperature, magnetic fields or electrical power. Thus, from one acquisition to the next one, if the environmental conditions are not the same, the MEMS behavior will not be the same.

In our investigations we will first consider the somewhat trivial (and less likely) case where the sensors of the same type (i.e. the gyroscopes or the accelerometers) have equal and time-invariant noise level within a system. Then, we allow variations to occur between sensors and in time. For such scenario our estimation will be based on a tool called generalized autoregressive conditional heteroskedasticity $(\mathrm{GARCH})$ which is a widespread approach dealing with heteroskedastic time series. We will show that this method offers an interesting tool to model the magnitude of the noise in the residuals of multi-IMU systems.

In this paper, we first recall the possible integration approaches for redundant IMUs with GPS data. Then, the noise reduction of redundant IMU systems is investigated. The theoretical expectations are compared to those obtained by an experiment with four MEMS-IMU triads. Finally, two methods that allow estimating the variation of the noise level through time are introduced. The first approach assumes that the variance is identical for all sensors and constant during a certain interval of time. Based on these assumptions, the classical definition of the variance is applied to the considered period of time. The second method is based on GARCH models and estimates the variance for the individual sensors, whose noise level can be different. Throughout the paper, the proposed approaches are supported by experimental findings with MEMS-IMUs in dynamic situations and the support of signals of higher quality for reference.

\section{Redundant IMUs in a loosely coupled GPS/INS system}

Redundancy in inertial navigation has been investigated in the past with higher order IMUs [4]. It can be generated at different levels [5].

Redundancy at system level: several GPS/INS components are formed and processed separately and the results are merged at the end. Although robust in terms of avoiding interdependences, this approach is not economical and remains sub-optimal with respect to the available information content.

Redundancy at sensor level: multiple IMUs are processed together or individually before applying the strapdown navigation and integration with GPS (figure 1).

- In a first method a synthetic IMU is formed from the available sensor data. In this situation, the modeling and estimation of the inertial errors cannot be separated for the individual sensors. Therefore, this simple approach is as well sub-optimal.

- In a second configuration, the processing can imply one or multiple navigation processors. In this so-called extended mechanization, the strapdown navigation equations are modified to accommodate error terms for all sensors [4].

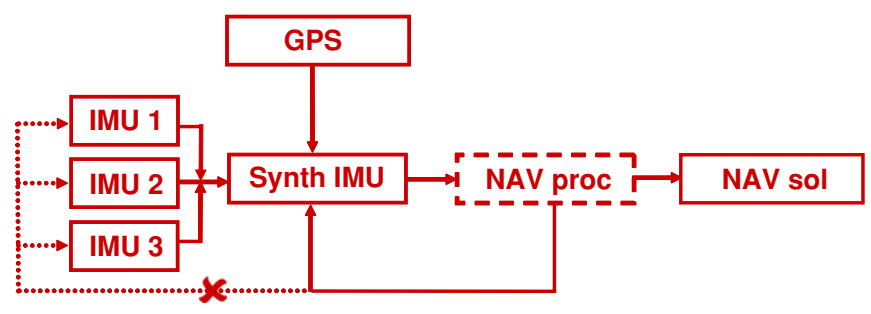

Figure 1. Principle of mechanization based on a synthetic IMU.

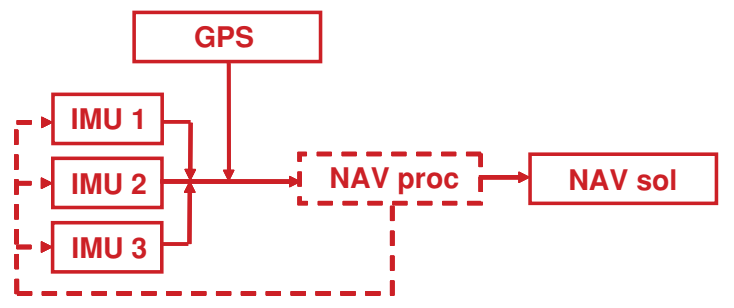

Figure 2. Principle of extended IMU mechanization.

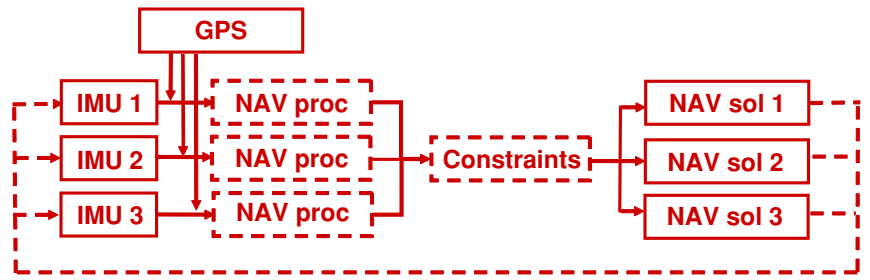

Figure 3. Principle of geometrically constrained IMU mechanization.

Hence, the separation of the systematic errors per sensor becomes possible in the GPS/INS filter (figure 2) [2].

- Finally, the so-called constrained IMU mechanization utilizes as many strapdown navigation processors as IMUs, while imposing the geometric condition between the obtained solutions (figure 3). Similarly to the previous technique, this method can be considered as optimal in terms of error modeling and estimation but requires more computational resources than the extended approach.

The three mentioned integration methods require knowledge of the noise level of the inertial sensors. In the case of 'synthetic IMU' approach, only compound information is required, while for the others the noise level should be distinguished for each sensor in the KF.

\section{Noise reduction}

From a theoretical point of view, the best estimate of the expected value $\hat{x}$ of $n$ independent measurements $x_{1}, \ldots, x_{n}$ (with their respective variances $\sigma_{1}^{2}, \ldots, \sigma_{n}^{2}$ ) can be computed as a weighted average of the measurements. Assuming homogeneous measurements (i.e. constant $\sigma_{i}$ ), its variance $\sigma_{\hat{x}}^{2}$ can be derived as [6]

$$
\sigma_{\hat{x}}^{2}=\sum_{i=1}^{n} w_{i}^{2} \sigma_{i}^{2}=\frac{\sigma_{x}^{2}}{n},
$$

where $w_{i}$ are the weighting factors and $\sigma_{x}=\sigma_{i} \forall i$. 


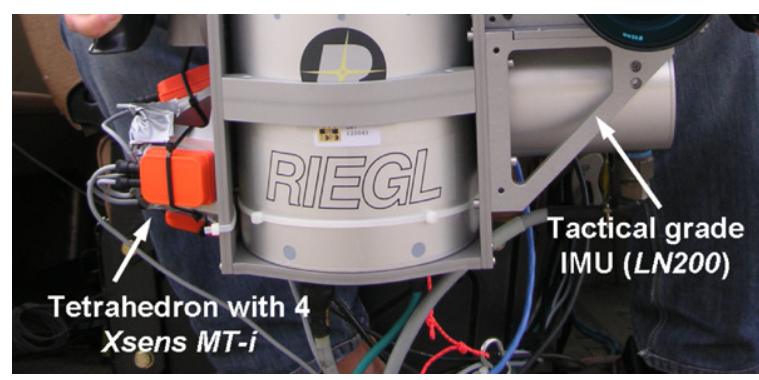

Figure 4. Skew-redundant MEMS-IMUs placed in a tetrahedron mounted together with a tactical grade IMU (Litton LN200).

To investigate the validity of the model proposed in equation (1), we conducted the following experiment. A regular tetrahedron consisting of four Xsens MT-i MEMSIMUs was mounted on a rigid structure together with a reference IMU (Litton LN200) embedded in a vehicle (figure 4).

Assuming independent measurements and according to equation (1), the noise affecting the best estimate $\hat{x}$ derived from measurements of four MEMS-IMUs is supposed to be two times lower than the noise of the individual MEMS-IMU. Hence, the expected noise reduction for such configuration is $50 \%$.

The theoretical reduction of the noise level was verified by comparing the differences between the MEMS-IMU measurements and their best estimate to the reference values. Thereafter, a parametric compensation was performed to remove systematic errors in the MEMS-IMU measurements. Thus, the remaining differences are assumed to be composed of white noise only. The averaged noise of the MEMS-IMU gyros was estimated to $0.0201 \mathrm{rad} \mathrm{s}^{-1}$, whereas the noise level of their best estimate amounts to $0.0103 \mathrm{rad} \mathrm{s}^{-1}$. Hence, the experimental noise reduction is approximately $48.6 \%$ which confirms the validity of the theoretical model. Figure 5 illustrates these results graphically.

Figure 6 shows the boxplot of residuals of the norm of four MEMS-IMUs and of synthetic IMU (i.e. the averaged norms of MEMS-IMU measurements). It can be observed that the variance of sensor MTi-14 is significantly different from the variance of the other sensors. Moreover, this sensor is biased and introduces in consequence a bias in the synthetic IMU. This example shows the limitation of the synthetic approach which gives equal weights to each sensor since their precisions are assumed to be equivalent. To reduce this limitation, section 5 proposes a method that weights measurements based on their estimated variance.

\section{Direct noise estimation-averaged volatilities}

In the first approach, we assumed that the variance remains constant around the point of interest in the interval containing $2 T+1$ measurements. Thus, the variance at $t$ can be written as

$$
\sigma_{t}^{2}(T)=\frac{1}{6 T n} \sum_{i=1}^{n} \sum_{k=t-T}^{t+T} \sum_{j=1}^{3}\left(x_{j_{i}}(k)-\bar{x}_{j}(t)\right)^{2},
$$

where $x_{j_{i}}(t)$ correspond to the ordinary least-squares residuals of the $i$ th sensor on the axis $j$ at the time $t, n$ is the number of redundant IMUs in the system and

$$
\bar{x}_{j}(t)=\frac{1}{(2 T+1) n} \sum_{i=1}^{n} \sum_{k=t-T}^{t+T} x_{j_{i}}(k) .
$$

The results of this approach are presented in figure 7 for various averaging windows of size $2 T+1$. Clearly, as the size of the averaging windows increases the estimated

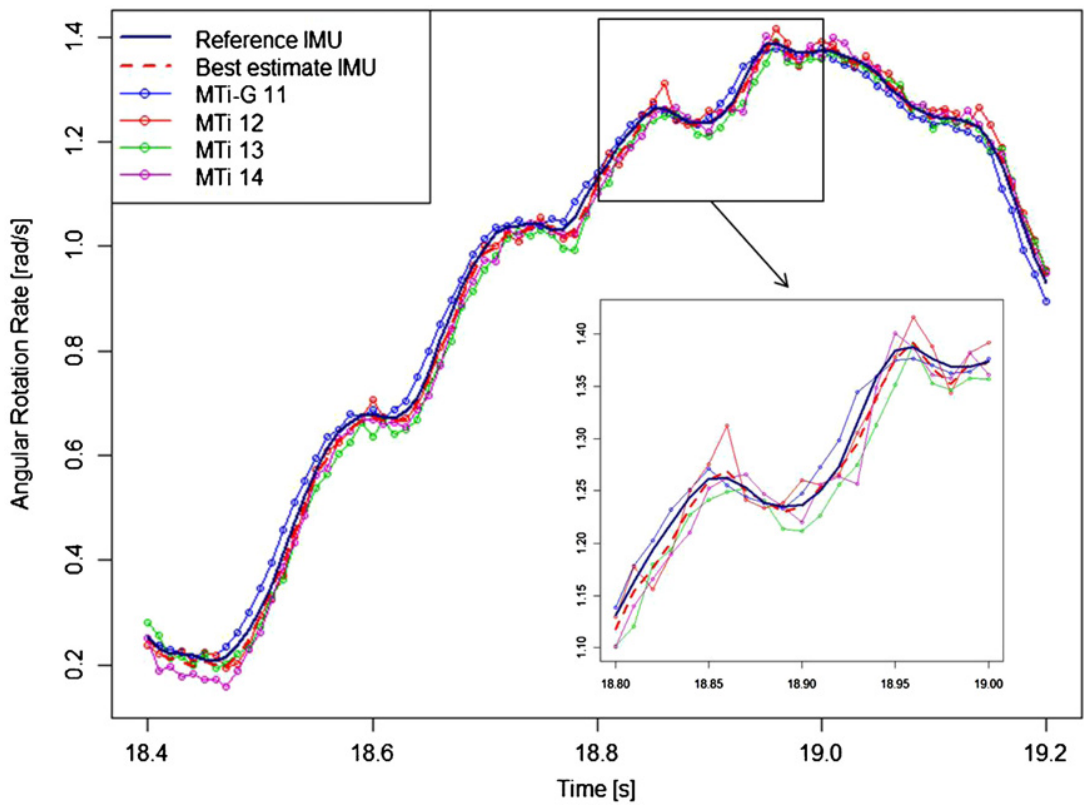

Figure 5. Norm of the angular rate measurements of four MEMS-IMUs (Xsens MTi) in comparison to the reference measurements from a tactical-grade IMU. 


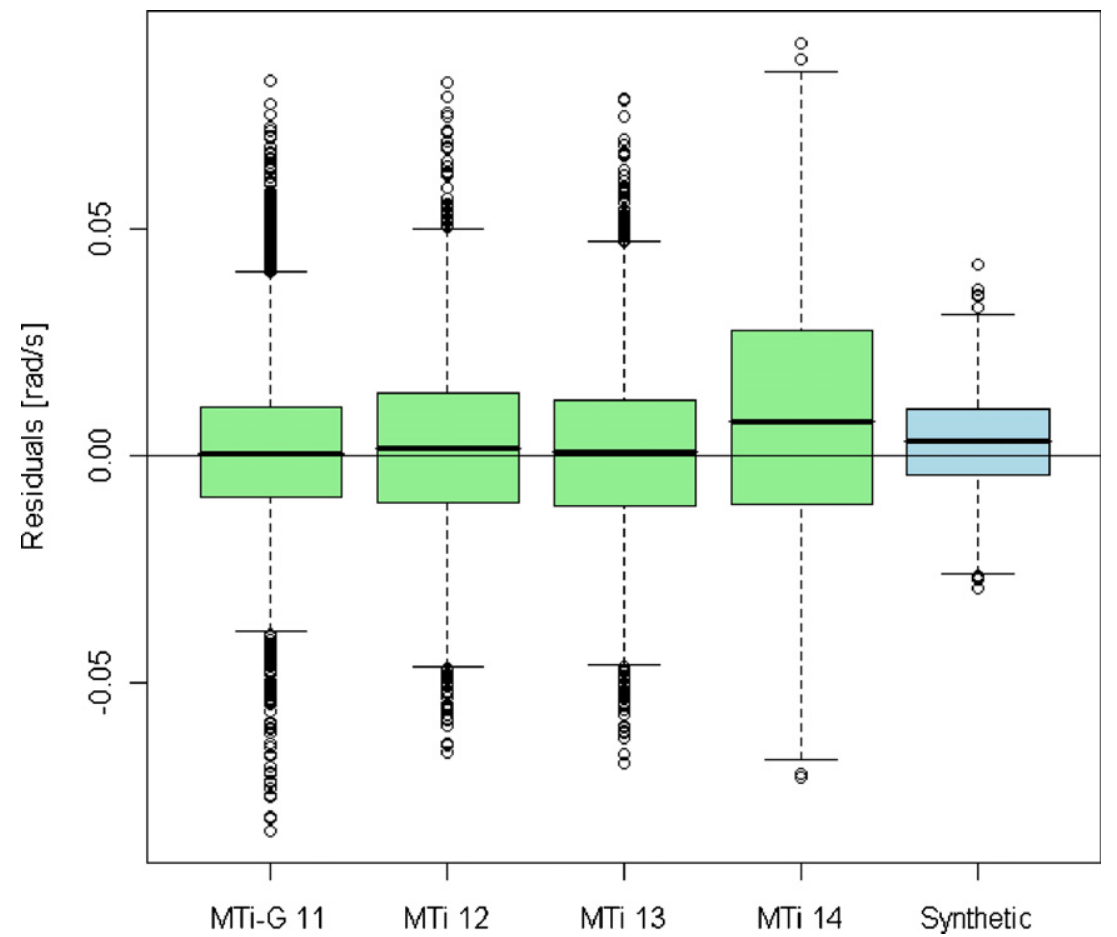

Figure 6. Boxplots of the residuals of the norm of four MEMS-IMUs and of the synthetic IMU.

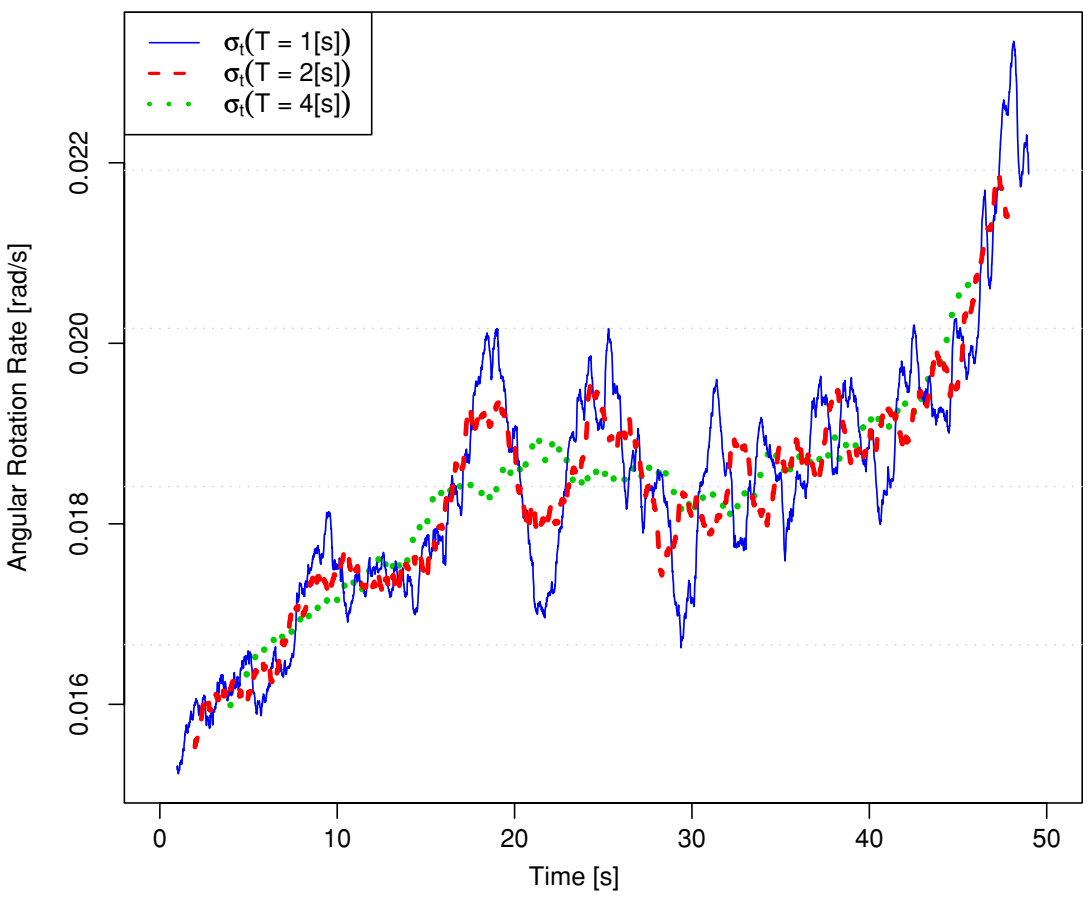

Figure 7. Noise levels estimated with various averaging windows.

variances are smoothed out. The performance of this approach is illustrated in figure 8 . It appears that the variance based on the least-squares residuals is underestimated compared to the one obtained using the residuals (i.e. errors) with respect to the reference signal. This result is expected and can be easily explained. Indeed, the estimated residuals are computed from $x_{j_{i}}(t)=\omega_{j_{i}}(t)-\frac{1}{n} \sum_{i=1}^{n} \omega_{j_{i}}(t)$ while the errors are computed as $\check{x}_{j_{i}}(t)=\omega_{j_{i}}(t)-\check{\omega}_{j_{i}}(t)$, where $\omega_{j_{i}}(t)$ represents the measurement of the $i$ th sensor on the axis $j$ at the time $t$ (after being projected into a suitable reference frame) and $\breve{\omega}_{j_{i}}(t)$ the reference measurement. It can be shown that

$$
\sum_{i=1}^{n} \check{x}_{j_{i}}^{2}(t)>\sum_{i=1}^{n} x_{j_{i}}^{2}(t), \quad \forall \check{\omega}_{j_{i}}(t) \neq \frac{1}{n} \sum_{i=1}^{n} \omega_{j_{i}}(t) .
$$

Indeed, the quadratic form $\sum_{i=1}^{n}\left(\omega_{j_{i}}(t)-a\right)^{2}$ is minimized when $a=\frac{1}{n} \sum_{i=1}^{n} \omega_{j_{i}}(t)$ (see the appendix for details). 


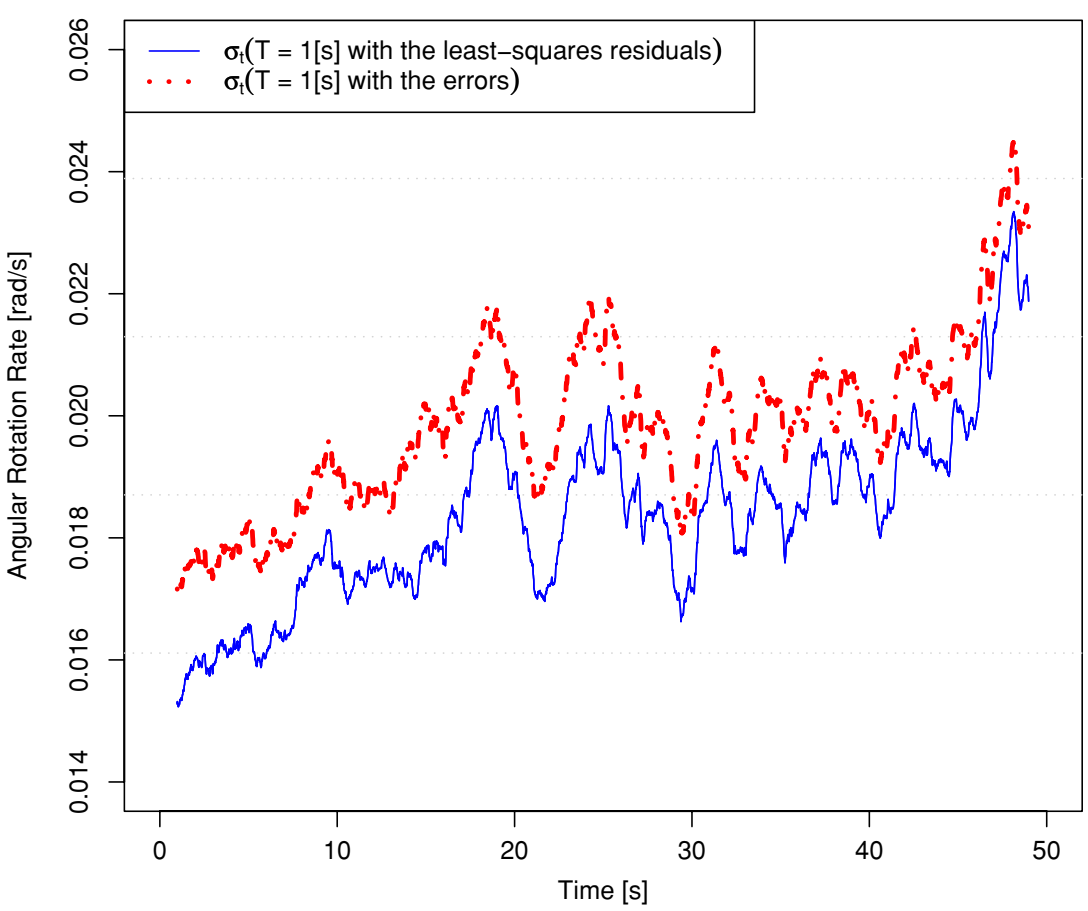

Figure 8. Comparison between the noise levels based on the least-squares residuals and with the errors (i.e. obtained with the reference signal).

It should be noted that this method is based on assumptions that may not correspond to reality. Firstly, it does not offer the possibility of estimating the variance of each sensor. Secondly, the assumption of homogeneous variance in the averaging window does not correspond to reality. In addition, this approach offers no guidance to choose an appropriate value of $T$. Finally, the estimate in this experience got relatively close to the reference signal (figure 8 ) only by a coincidence of the averaging process. This case cannot be generalized as the noise level can vary between sensors. The following approach will allow treating a more realistic scenario of the noise levels.

\section{Direct noise estimation-GARCH}

The second method for direct noise estimation is based on the aforementioned GARCH model. This model was originally developed in the 1980s and applied in economics and in finance. Indeed, the same problem of estimating the variance in a series through time often arises in financial applications. This is typically the case when the returns on an asset or portfolio are considered. The variance of these returns represents the risk level. Looking at financial data suggests that sometime periods are riskier than others, i.e. the expected variance of the error terms, at some moments, is greater than at others. Moreover, these risky periods are not scattered randomly across time but are rather found in clusters. In other words, after a large (small) price change a large (small) price change tends to occur. It is assumed that positive and negative error terms have a symmetric effect on the volatility. Such an assumption appears reasonable when dealing with IMU but is often violated when applied to financial time series [7]. Thus, the returns are not independent since their variability depends on recent changes of price [7]. In the context of MEMSIMU this may represent cases when the uncompensated effects start to influence sensor performance (exceeded temperature range or dynamics, vibrations, etc). The GARCH model was introduced in [8] as a generalization of ARCH models [9] that provide a measurement of the volatility across time.

$\operatorname{A~} \operatorname{GARCH}(p, q)$ model is typically specified by two numbers $p$ and $q$. The first one refers to the number of autoregressive lags in the model, while the second refers to the number of lags included in the moving average component of a variable. Such models rely on the assumption that the volatility can be written as a linear combination of previous terms and previously estimated volatilities. Therefore, it is employed to estimate the volatility through the time of a series. It also offers the possibility of forecasting variances based on historical values.

GARCH models are based on an assumption that the time series of interest is unpredictable. Let $\Omega_{t}$ denote the information set at time $t$. Then, the best prediction at $t$ for $t+1$ is the conditional expectation $E\left[x_{t+1} \mid \Omega_{t}\right]$ based on the information set at time $t$. The time series $x_{t}$ is called unpredictable if the best prediction of $x_{t+1}$ at $t$ is simply its unconditional mean [7], i.e.

$$
E\left[x_{t+1} \mid \Omega_{t}\right]=E\left[x_{t+1}\right] .
$$

By definition, an unpredictable time series is only composed of white noise since introducing any colored noise would create some form of autocorrelation in time that could be predicted.

The GARCH model also relies on the idea that in an unpredictable time series volatility tends to form clusters. This implies that squared values of the series (i.e. $x_{t}^{2}$ ) are positively 

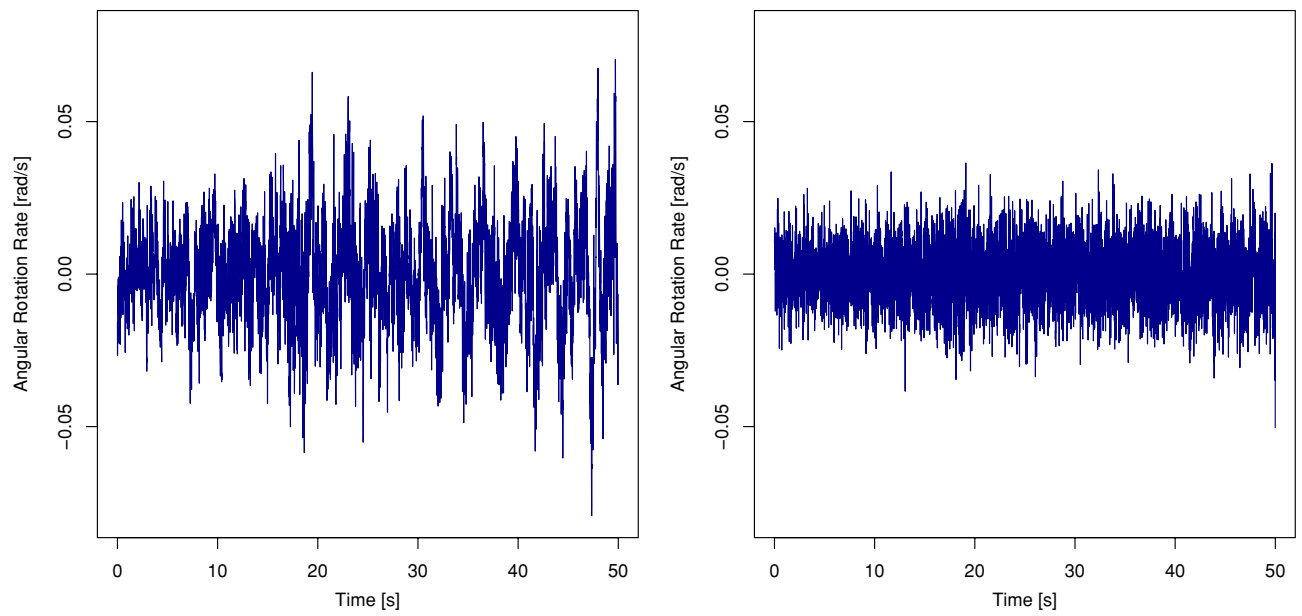

Figure 9. Least-squares residuals of the Xsens MTi-G on the $x$-axis (left). ARMA residuals of the same sensor (right).
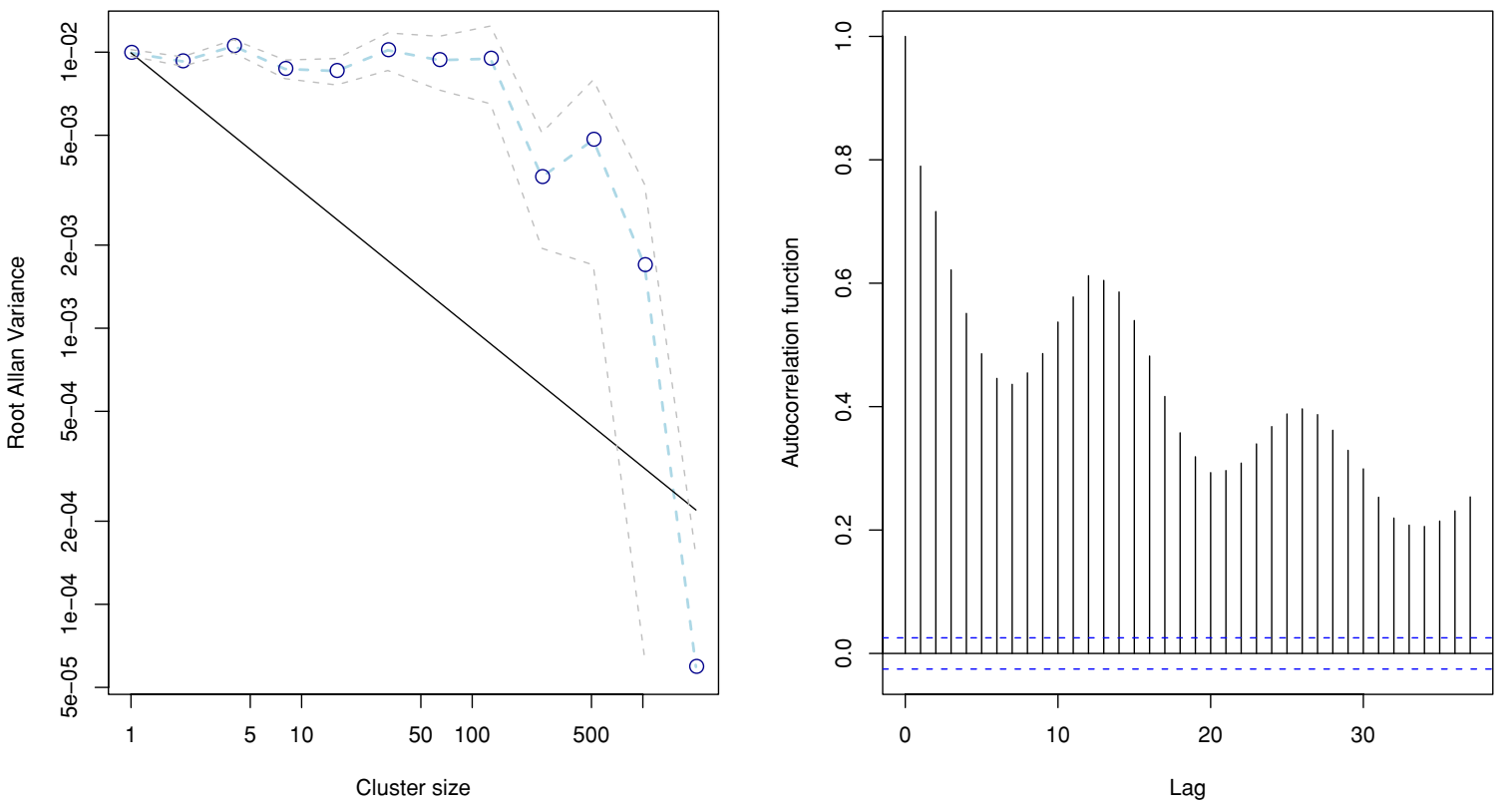

Figure 10. Results of the Allan variance performed on the residuals of the Xsens MTi-G on the $x$-axis (left). The autocorrelation function of the same residuals (right).

correlated [7]. Thus, the time series $x_{t}$ is conditionally heteroskedastic, i.e.

$$
\operatorname{Var}\left[x_{t+1} \mid \Omega_{t}\right] \neq \operatorname{Var}\left[x_{t+1}\right] \text {. }
$$

Formally a (strong) $\operatorname{GARCH}(p, q)$ model is defined as follows: the process $x_{t}, t \in \mathbb{Z}$, is $\operatorname{GARCH}(p, q)$ if $E\left[x_{t} \mid \Omega_{t-1}\right]=E\left[x_{t}\right]=0$,

$$
\sigma_{t}^{2}=\alpha_{0}+\sum_{i=1}^{p} \alpha_{i} x_{t-i}^{2}+\sum_{i=1}^{q} \beta_{i} \sigma_{t-i}^{2}
$$

and $\operatorname{Var}\left[x_{t} \mid \Omega_{t-1}\right]=\sigma_{t}^{2}$. Assuming $x_{t}$ to be normally distributed it implies that $Z_{t}=\frac{x_{t}}{\sigma_{t}} \sim N(0,1)$. Moreover, it follows that the maximum likelihood estimator $\hat{\phi}$ is consistent and asymptotically normally distributed:

$$
\sqrt{n}(\hat{\boldsymbol{\phi}}-\phi) \stackrel{D}{\rightarrow} N_{p+q+1}\left(\mathbf{0}, \boldsymbol{J}^{-1}\right),
$$

where $\phi=\left(\alpha_{0}, \alpha_{1}, \ldots, \alpha_{p}, \beta_{1}, \ldots, \beta_{q},\right)^{T}$ is the parameter vector. $\boldsymbol{J}$ represents the Fisher information matrix defined by

$$
\boldsymbol{J}=E\left[-\frac{\partial^{2} \ell(\boldsymbol{\phi}, \boldsymbol{x})}{\partial \phi \partial \phi^{T}}\right],
$$

where $\ell(\phi, x)$ is the log-likelihood function. Thus, equation (8) allows us to compute confidence regions for $\phi$ and $\sigma_{t}$. Moreover, the parameter significativity can then be tested by a significance Student test.

In practice, the assumption of unpredictability has to be verified before applying GARCH models. Although it is verified in most financial applications, it is clearly not the case with MEMS-IMUs. Indeed, it has been demonstrated in $[3,10,11]$ that the errors of MEMS-IMUs are not only composed of white noise but also of various types of colored noise. Figure 9 (left) shows the residuals of an Xsens MTi-G on the $x$-axis; these clearly appear autocorrelated. Additionally, figure 10 presents the results of the Allan variance performed 

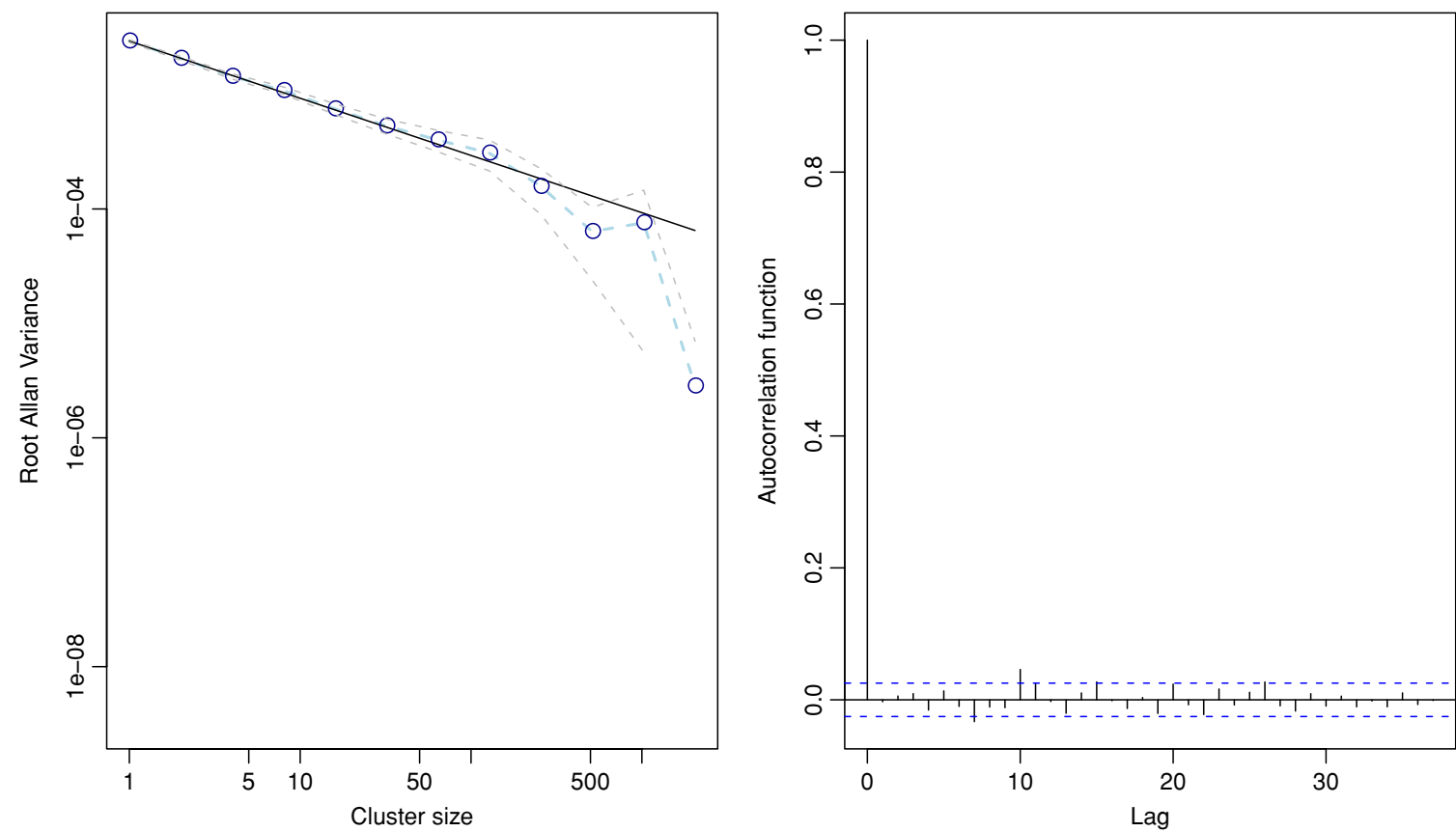

Figure 11. Results of the Allan variance performed on the ARMA residuals of the Xsens MTi-G on the $x$-axis (left). Autocorrelation function of the same residuals (right).

on these residuals as well as the results of the autocorrelation function. It can be observed that the Allan variance as well as the autocorrelation function confirms that these residuals are autocorrelated.

For this reason, we employed autoregressive moving average (ARMA) models to remove the part of the noise that is autocorrelated [12-14]. The $\operatorname{ARMA}(p, q)$ model is defined as

$$
x_{t}=v+\sum_{i=1}^{p} \alpha_{i} x_{t-i}+\sum_{j=1}^{q} \beta_{j} \epsilon_{t-j}+\epsilon_{t} .
$$

Under the distribution assumption that $\epsilon_{t} \sim N\left(0, \sigma^{2}\right)$ and some additional technical assumptions (see for example [7]) the maximum likelihood estimator $\hat{\boldsymbol{\theta}}$ is consistent, asymptotically efficient and has an asymptotic normal distribution given by

$$
\sqrt{n}(\hat{\boldsymbol{\theta}}-\boldsymbol{\theta}) \stackrel{D}{\rightarrow} N_{p+q+1}\left(\mathbf{0}, \boldsymbol{J}^{-1}\right),
$$

where $\boldsymbol{\theta}=\left(\nu, \alpha_{1}, \ldots, \alpha_{p}, \beta_{1}, \ldots, \beta_{q}, \sigma^{2}\right)^{T}$ is the parameter vector and $\boldsymbol{J}$ is the Fisher information matrix. As for the GARCH models, it follows that equation (11) can be used to derive confidence regions for $\boldsymbol{\theta}$ and $\epsilon_{t}$.

The selection of the appropriate $p$ and $q$ was realized based on the Akaike information criterion (AIC) [15]. The AIC does not test models against each other in the classical sense of hypothesis testing. But given a data set, several competing models may be ranked according to their AIC, with the one having the lowest AIC being the best. Such an approach revealed, for example, that the residuals of the Xsens MTi-G IMU on the $x$-axis are best modeled by an $\operatorname{ARMA}(2,2)$. In this case, it implies that $E\left[x_{1_{1}}(t) \mid \Omega_{t-1}\right]=$ $1.67 x_{1_{1}}(t-1)-0.67 x_{1_{1}}(t-2)-1.09 \epsilon_{1_{1}}(t-1)+0.16 \epsilon_{1_{1}}(t-2)$.
Table 1. Estimation results for the estimation of the ARMA parameters of the Xsens MTi-G IMU's residuals on the $x$-axis. The ' $t$-value' and the ' $p$-value' are obtained by a Student test aiming to assess the significance of the parameters. The ' $p$-value' is the probability of obtaining a test statistic at least as extreme as the one that was actually observed, assuming that the null hypothesis $H_{0}$ (i.e. the considered parameter is not significant) is true. The ' $t$-value' is the statistic of the test.

\begin{tabular}{lclrl}
\hline & Estimate & Standard deviation & $t$-value & $p$-value \\
\hline$\alpha_{1}$ & 1.666 & $2.12 \times 10^{-2}$ & 78.57 & $\approx 0$ \\
$\alpha_{2}$ & -0.671 & $2.06 \times 10^{-2}$ & -32.61 & $\approx 0$ \\
$\beta_{1}$ & -1.086 & $2.32 \times 10^{-2}$ & -46.72 & $\approx 0$ \\
$\beta_{2}$ & 0.160 & $1.76 \times 10^{-2}$ & 9.11 & $\approx 0$ \\
$\nu$ & $-3.5 \times 10^{-7}$ & $1.23 \times 10^{-5}$ & -0.029 & 0.977 \\
\hline
\end{tabular}

The estimated precision of these parameters is summarized in table 1 . It shows that all the parameters except $v$ are highly significant.

Figure 9 (right) shows the residuals of the ARMA model of the MTi-G IMU on the $x$-axis. In addition, figure 11 depicts the results of the Allan variance performed on these residuals as well as the results of the autocorrelation function. These figures confirm that the residuals of the ARMA are distributed randomly, i.e are unpredictable; thus, $E\left[\epsilon_{t} \mid \Omega_{t-1}\right]=E\left[\epsilon_{t}\right]=$ 0 . However, the residuals of the ARMA models are not homoskedastic (i.e. if the considered sequence of random variables have the same finite variance). This is illustrated in figure 12. The estimation of the variances is then realized with the help of GARCH models.

The selection procedure of GARCH models relies on the idea that $Z_{t}$ (as defined in equation (7)) should be such that $E\left[Z_{t}\right]=0$ and $\operatorname{Var}\left[Z_{t}\right]=1$ if the GARCH model is correct. Thus, various models of growing complexity 

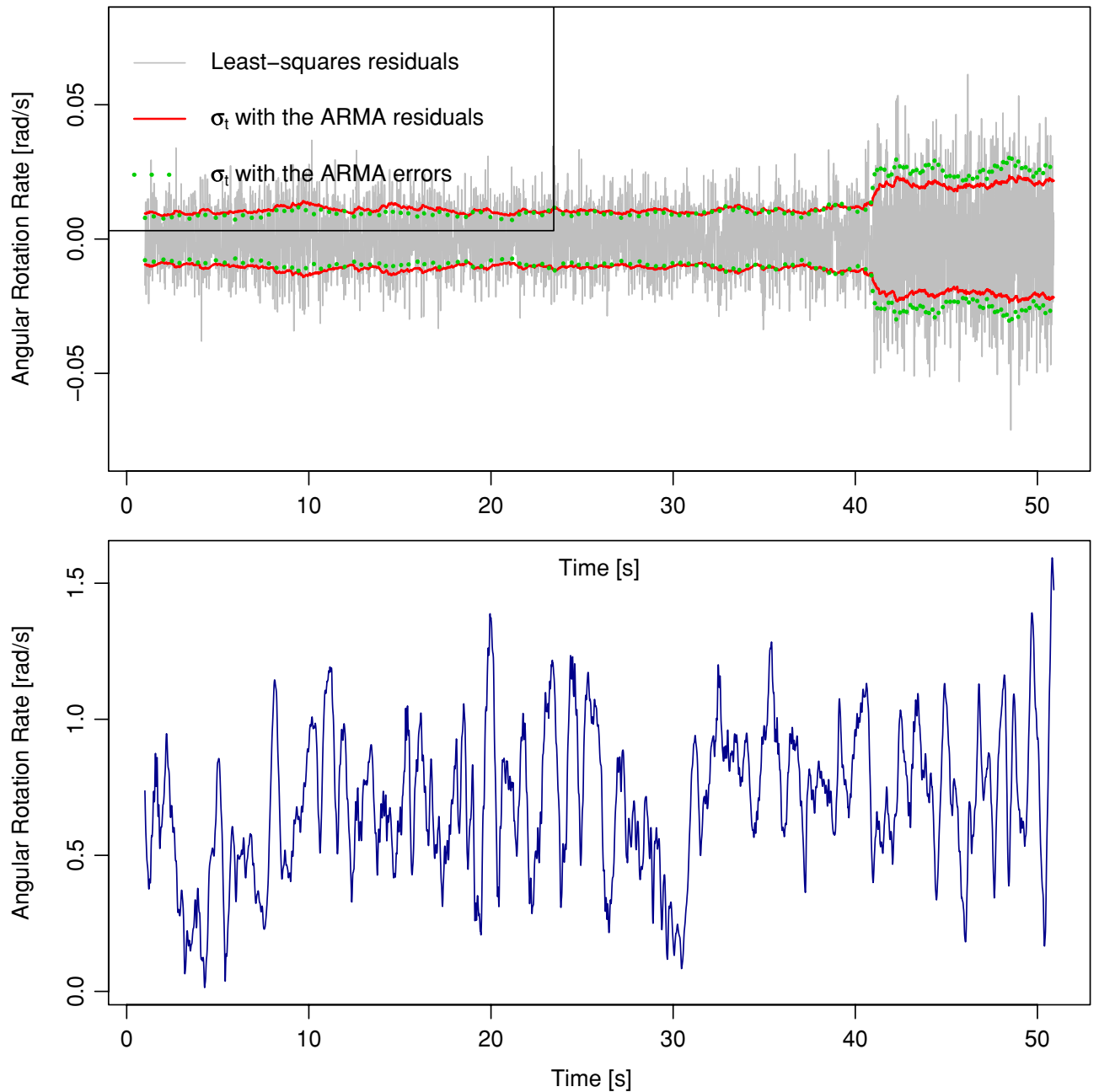

Figure 12. Variances computed with the ARMA errors and the ARMA residuals of the Xsens MTi-G on the $z$-axis (above). Norm of the angular rate measurements of the reference IMU (below).

(i.e. with $p$ and $q$ increasing) were estimated until a test revealed that no trace of heteroskedasticity is remaining in $Z_{t}$. Indeed, [9] derived such a test based on the Lagrange multiplier principle. The statistics of this test converges to a $\chi^{2}$ distribution under the null hypothesis that the considered time series consists of independent and identically distributed Gaussian disturbances [16]. The model selection process revealed that a $\operatorname{GARCH}(1,1)$ was appropriate to describe the volatility in the considered MEMS-IMUs.

For example, the variance of the Xsens MTi-G IMU's residuals on the $z$-axis is estimated by $\sigma_{t}^{2}=0.0175 x_{t-1}^{2}+$ $0.982 \sigma_{t-1}^{2}$. The estimated precision of these parameters is summarized in table 2 . It shows that all the parameters except $\alpha_{0}$ are highly significant.

Figure 12 compares the estimations of the volatility based on the estimated ARMA residuals and the ARMA errors (i.e. the difference between the MEMS measurements after the ARMA correction and the LN200 measurements) on the $z$-axis of the Xsens MTi-G.

The good correspondence between both plots indicates that the volatility estimation by the GARCH method is relatively accurate. Moreover, the volatility estimation using
Table 2. Estimation results for the estimation of the GARCH parameters of the Xsens MTi-G IMU's residuals on the $z$-axis.

\begin{tabular}{lllrl}
\hline & Estimate & Standard deviation & $t$-value & $p$-value \\
\hline$\alpha_{0}$ & $1.3 \times 10^{-7}$ & $1.1 \times 10^{-7}$ & 1.15 & 0.25 \\
$\alpha_{1}$ & $1.75 \times 10^{-2}$ & $2.7 \times 10^{-3}$ & 6.45 & $\approx 0$ \\
$\beta_{1}$ & 0.98 & $3.0 \times 10^{-3}$ & 326.85 & $\approx 0$ \\
\hline
\end{tabular}

this approach enables weighting the measurements according to their estimated variances. Thus, we defined the corrected version of the synthetic IMU as

$$
\hat{\omega}_{j t}=\frac{\sum_{i=1}^{n} \sigma_{j_{i}}^{-2}(t)\left(\omega_{j_{i}}(t)-E\left[x_{j_{i}}(t)\right]\right)}{\sum_{i=1}^{n} \sigma_{j_{i}}^{-2}(t)},
$$

where $\sigma_{j_{i}}^{2}(t)$ is the variance estimated with a GARCH model of the measurement error of the $i$ th sensor on the $j$ th axis at time $t$, i.e. $\sigma_{j_{i}}^{-2}(t)=\operatorname{Var}\left[\omega_{j_{i}}(t)-E\left[x_{j_{i}}(t)\right] \mid \Omega_{t-1}\right]$.

The resulting noise reduction of this weighting process improves the results presented in section 3 (figure 13). Indeed, the MSE of the synthetic IMU is equal to $11.26 \times 10^{-5}$ while the MSE of the corrected synthetic equals $7.90 \times 10^{-5}$. Thus, 


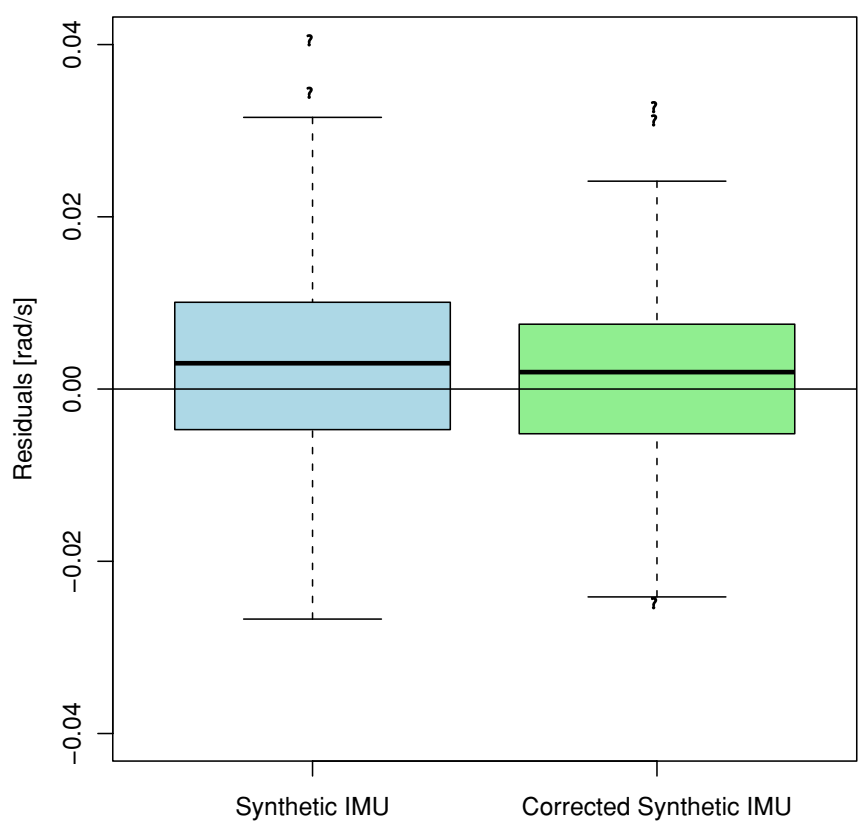

Figure 13. Boxplots of the residuals of the norm of the synthetic and of the weighted synthetic IMU.

the efficiency of the corrected synthetic compared to the classical approach is approximately $70.2 \%$. Moreover, the noise level of the corrected IMU amounts to $0.0088 \mathrm{rad} \mathrm{s}^{-1}$. This implies a reduction of $14.6 \%$ compared to the synthetic IMU and an overall noise reduction of $56.2 \%$. Note that this is more than $50 \%$ provided by the theory. This difference can be explained by the inhomogeneity of the variances of the sensors.

\section{Discussion}

A clear comparison between the GARCH method and the 'averaged volatilities' method is difficult to perform since the exact volatility of the system is unknown and can only be estimated using one of the proposed approaches. However, we believe that the GARCH approach is more appropriate to describe the noise variations of MEMS-IMUs. On the other hand, the first approach is simple; hence, it could be easily automated and included within the GPS/INS integration. Nevertheless, the underlying assumption that the noise level is constant during a certain interval of time and equal for all sensors may not be correct and is, moreover, difficult to verify experimentally. As this approach does not offer the possibility of estimating the variance at the sensor level, the weighted version of the synthetic IMU cannot be computed (or the diagonal of the covariance matrix used in the extended mechanization). In other words, this method estimates only the mean variation of the noise level across sensors. The GARCH approach is, on the other hand, more sophisticated. Although this method requires more computational effort, it seems to have a finer resolution and is more precise in reflecting the reality. This can be seen in figure 14 which compares graphically the evolution of noise level in time estimated by both methods. It can be observed that the volatility estimated with the GARCH method seems to be appropriate to model the heteroskedasticity of the residuals. On the other hand, the results obtained with the averaged volatilities are not satisfactory as they only describe the average heteroskedasticity among all sensors. In addition, the variance estimation with this method might be slightly

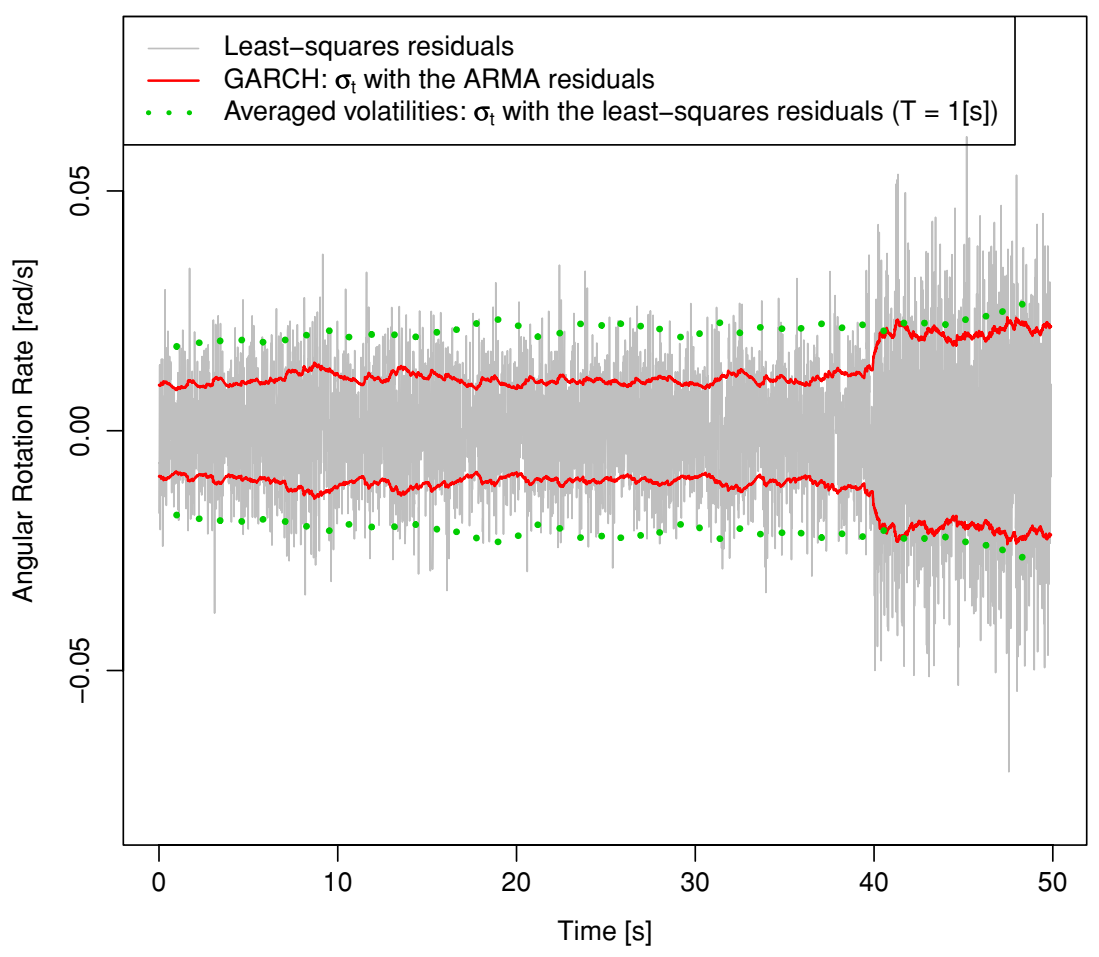

Figure 14. Comparison between the noise level estimated with the 'averaged volatilities' and with the GARCH methodology of the Xsens MTi-G on the $z$-axis. 
Table 3. Summary of the main differences between the averaged volatilities approach and the GARCH method. ' + ' represents an advantage while '-' an inconvenience.

\begin{tabular}{|c|c|c|c|}
\hline & Moving average estimator & & GARCH (+ARMA) \\
\hline+ & Simple and easily automated & $-/+$ & $\begin{array}{l}\text { Require large effort to automate parameter } \\
\text { estimation and model selection }\end{array}$ \\
\hline+ & Low computational effort & - & Higher computational effort \\
\hline- & The volatility of each sensor cannot be modeled & + & The volatility of each sensor is modeled \\
\hline$-/+$ & $\begin{array}{l}\text { Non-robust estimation in principle. } \\
\text { Extension to robust estimator easy to implement }\end{array}$ & - & $\begin{array}{l}\text { Non-robust estimation in principle. } \\
\text { Extension to robust estimation complex and } \\
\text { computationally intensive [17] }\end{array}$ \\
\hline- & 'Predictable' part of the errors is ignored & + & 'Predictable' part of the errors is modeled (ARMA) \\
\hline
\end{tabular}

Table 4. Standard deviations $\left(\mathrm{rad} \mathrm{s}^{-1}\right)$ of the errors of the norm of the synthetic or of the weighted synthetic IMU.

\begin{tabular}{lccc}
\hline & $a=0$ & $a=5$ & $a=10$ \\
\hline Synthetic IMU & 0.0103 & 0.0156 & 0.0262 \\
Weighted synthetic IMU & 0.0088 & 0.0104 & 0.0112 \\
\hline
\end{tabular}

overestimated since the 'predictable' part of the noise is not properly modeled. Table 3 summarizes the main differences between the two approaches.

The benefits of the GARCH approach might be larger when

- the noise level varies to a greater extent in time,

- the stochastic input to the KF substantially differs from the reality,

- the precision levels are very different among the sensors.

To illustrate the latter case, additional noise was added to the measurements of the Xsens MTi-G on the $x$-axis. The introduced perturbation of measurement can be expressed as

$$
\tilde{\omega}_{1_{1}}(t)=\omega_{1_{1}}(t)+\sin \left(\frac{6 \pi t}{t_{\max }}\right) W
$$

where $t_{\max }$ is the last value of the time vector, $W$ is random variable independent and identically distributed as $a N\left(0, \operatorname{var}\left[\omega_{1_{1}}(t)\right]\right)$ with $a$ representing an arbitrary perturbation constant. The standard deviations of the errors of the norm for the synthetic and the weighted synthetic IMU are given in table 4 for three values of $a$. Clearly, as $a$ increases the standard deviation of the weighted synthetic IMU is affected only slightly while the standard deviation of the synthetic IMU with equal weighting substantially increases.

This shows that the employment of direct noise estimation increases the noise reduction of multi-IMU systems as well as its robustness. In addition, other objectives were targeted with direct noise estimation.

Firstly, direct noise estimation reduces user interaction and user knowledge (of the algorithms and sensors) requirements which facilitates the automation of the processing. Secondly, noise variations (especially from MEMS accelerometers) can be determined online which may improve filter stability and maintain navigation performance. Lastly, direct noise estimation can improve the fault detection and isolation (FDI) performance $[1,2]$ which also depends on the correctness of the noise model.
Our future investigations will concentrate on assessing the performance improvement of the navigation states with the GARCH approach with the different mechanization methods as well as on better understanding of the influence of vehicle dynamics on sensor noise. In addition, the multivariate GARCH model will also be investigated to model the full covariance matrix of the measurements. We believe that this could further improve the navigation performances.

\section{Conclusion}

In this paper, we investigated the noise reduction resulting from redundant measurements and the estimation of the noise level during the processing. We have shown that the assumption of independent measurement is reasonable when employing redundant MEMS-IMUs of the same type. Moreover, we presented two methods that assess the volatility of redundant IMU systems. Despite their differences, both methods appear appropriate to model noise variations. The first approach is adapted from the classical definition of the variance and relies on assumptions that may not be statistically correct. However, this method is simple and could easily be integrated as a part of the GPS/INS integration process. In contrast, the GARCH approach is far more sophisticated as it is able to describe variations of the noise level in a more accurate manner. Hence, this approach enables us to compute a corrected version of the classical synthetic IMU by introducing measurement weights. Experimental results have shown that the noise level of the weighted synthetic is reduced by $56 \%$ while it is only $48 \%$ by the classical approach. Further simulations revealed that the relatively small differences between both methods (14.6\%) can be considerably larger (up to $100 \%$ ) when the noise level of one of the IMUs differs substantially from the others.

\section{Acknowledgments}

The authors would like to thank Pierre Bajgrowicz for his help on GARCH models as well as Professor Maria-Pia VictoriaFeser for sharing her thoughts and ideas. The Institute of Geomatics contribution was funded by the GENIA research project of the 'Programa Nacional del Espacio', Spanish Ministry of Education and Science, ESP2005-07599. Finally, the authors wish to thank two referees for their valuable comments that improved this paper. 


\section{Appendix}

The quadratic form $\sum_{i=1}^{n}\left(\omega_{j_{i}}(t)-a\right)^{2}$ is minimized when $a=\frac{1}{n} \sum_{i=1}^{n} \omega_{j_{i}}(t)$, since

$$
\begin{aligned}
& \sum_{i=1}^{n}\left(\omega_{j_{i}}(t)-a\right)^{2}=\sum_{i=1}^{n}\left(\omega_{j_{i}}(t)^{2}+a^{2}-2 a \omega_{j_{i}}(t)\right) \\
& \frac{\partial}{\partial a} \sum_{i=1}^{n}\left(\omega_{j_{i}}(t)-a\right)^{2}=\sum_{i=1}^{n}\left(2 a-2 \omega_{j_{i}}(t)\right) \\
& \frac{\partial}{\partial a} \sum_{i=1}^{n}\left(\omega_{j_{i}}(t)-\hat{a}\right)^{2}=0 \Rightarrow \hat{a}=\frac{1}{n} \sum_{i=1}^{n} \omega_{j_{i}}(t) .
\end{aligned}
$$

\section{References}

[1] Waegli A 2009 Trajectory determination and analysis in sports by satellite and inertial navigation PhD Thesis 4288, EPFL

[2] Waegli A, Guerrier S and Skaloud J 2008 Redundant MEMS IMU integrated with GPS for performance assessment in sports Proc. IEEE/ION PLANS 2008 (Monterey, $C A$ )

[3] Guerrier S 2009 Improving accuracy with multiple sensors: study of redundant MEMS IMU/GPS configurations Proc. ION GNSS 2009 (Savannah, GA, USA)

[4] Colomina I, Giménez M, Rosales J J, Wis M, Gómez A and Miguelsanz P 2004 Redundant IMUs for precise trajectory determination Proc. XXth ISPRS Congress (Istanbul, Turkey, Working Group I/5)
[5] Allerton D J and Jia H 2005 A review of multisensor fusion methodologies for aircraft navigation systems J. Navig. 58 405-17

[6] Titterton D H and Weston J L 1997 Strapdown Inertial Navigation Technology (London: Peter Peregrinus)

[7] Franke J, Härdle W and Hafner C M 2000 Statistics of Financial Markets (Berlin: Springer)

[8] Bollerslev T 1986 Generalized autoregressive conditional heteroskedasticity J. Econometrics 31 307-27

[9] Engle R F 1982 Autoregressive conditional heteroscedasticity with estimates of variance of United Kingdom inflation Econometrica 50 987-1008

[10] El-Sheimy N, Hou H and Niu X 2008 Analysis and modeling of inertial sensors using allan variance IEEE Trans. Instrum. Meas. 57 140-9

[11] Hou H 2004 Modeling inertial sensors errors using Allan variance Master's Thesis Geomatics Engineering, University of Calgary

[12] Nassar S 2003 Improving the inertial navigation system (INS) error model for INS and INS/DGPS applications $P h D$ Thesis University of Calgary

[13] Nassar S, Schwarz K-P, El-Sheimy N and Noureldin A 2004 Modeling inertial sensor errors using autoregressive (AR) models Navig.: J. Inst. Navig. 51 259-68

[14] Park M and Gao Y 2006 Error analysis and stochastic modeling of low-cost MEMS accelerometer J. Intell. Robot. Syst. 46 27-41

[15] Greene W H 2003 Econometric Analysis (Englewood Cliffs, NJ: Prentice-Hall)

[16] Hamilton J D 1994 Time Series Analysis (Princeton, NJ: Princeton University Press)

[17] Mancini L, Trojani F and Ronchetti E 2005 Optimal conditionally unbiased bounded-influence inference in dynamic location and scale models J. Am. Stat. Assoc. $100628-41$ 